\title{
Unstable moyamoya disease: clinical features and impact on perioperative ischemic complications
}

\author{
Takeshi Funaki, MD,1 Jun C. Takahashi, MD, PhD, ${ }^{1}$ Yasushi Takagi, MD, PhD,1 \\ Takayuki Kikuchi, MD, PhD, ${ }^{1}$ Kazumichi Yoshida, MD, PhD, ${ }^{1}$ Takafumi Mitsuhara, MD, PhD, ${ }^{1}$ \\ Hiroharu Kataoka, MD, PhD, ${ }^{2}$ Tomohisa Okada, MD, PhD, ${ }^{3}$ Yasutaka Fushimi, MD, PhD, ${ }^{3}$ \\ and Susumu Miyamoto, MD, PhD'
}

Departments of ${ }^{1}$ Neurosurgery and ${ }^{3}$ Diagnostic Imaging and Nuclear Medicine, Kyoto University Graduate School of Medicine, Kyoto; and 'Department of Neurosurgery, National Cerebral and Cardiovascular Center, Osaka, Japan

OBJECT Unstable moyamoya disease, reasonably defined as cases exhibiting either rapid disease progression or repeated ischemic stroke, represents a challenge in the treatment of moyamoya disease. Despite its overall efficacy, direct bypass for such unstable disease remains controversial in terms of safety. This study aims to reveal factors associated with unstable disease and to assess its impact on postoperative silent or symptomatic ischemic lesions.

METHODS This retrospective cohort study included both pediatric and adult patients with moyamoya disease who had undergone 140 consecutive direct bypass procedures at Kyoto University Hospital. "Unstable moyamoya disease" was defined as either the rapid progression of a steno-occlusive lesion or repeat ischemic stroke, either occurring within 6 months of surgery. The extent of progression was determined through a comparison of the findings between 2 different MR angiography sessions performed before surgery. The clinical variables of the stable and unstable disease groups were compared, and the association between unstable disease and postoperative diffusion-weighted imaging (DWI)-detected lesion was assessed through univariate and multivariate analyses with generalized estimating equations.

RESULTS Of 134 direct bypass procedures performed after patients had undergone at least 2 sessions of MR angiography, 24 (17.9\%) were classified as cases of unstable disease. Age younger than 3 years $(p=0.029)$, underlying disease causing moyamoya syndrome $(p=0.049)$, and radiographic evidence of infarction $(p=0.030)$ were identified as factors associated with unstable disease. Postoperative DWI-defined lesions were detected after 13 of 140 procedures (9.3\%), although only 4 lesions $(2.9 \%)$ could be classified as a permanent complication. The incidence of postoperative DWI-detected lesions in the unstable group was notable at 33.3\% (8 of 24). Univariate analysis revealed that unstable disease $(p<0.001)$, underlying disease $(p=0.028)$, and recent stroke $(p=0.012)$ were factors associated with DWI-detected lesions. Unstable disease remained statistically significant after adjustment for covariates in both the primary and sensitivity analyses (primary analysis: OR 6.62 [95\% Cl 1.79-24.5]; sensitivity analysis: OR 5.36 [95\% Cl 1.47-19.6]).

CONCLUSIONS Unstable moyamoya disease, more prevalent in younger patients and those with underlying disease, is a possible risk factor for perioperative ischemic complications. Recognition of unstable moyamoya disease may contribute to an improved surgical result through focused perioperative management based on appropriate surgical risk stratification.

http://thejns.org/doi/abs/10.3171/2014.10.JNS14231

KEY WORDS moyamoya disease; rapid progression; cerebral revascularization; intraoperative complication; vascular disorders

A MAJOR characteristic of moyamoya disease is the chronic progression of stenosis in the terminal portion of the internal carotid artery (ICA) ${ }^{24}$ The rate of this progression varies by patient. Angiographic progression can occur in one-half of pediatric patients and one-quarter of adult patients, ${ }^{19,21}$ while angiographic findings in the remaining patients are stable for years. Some patients demonstrate extremely rapid progression from disease onset, resulting in poor outcome. ${ }^{4,17,18}$ Fujiwara et al. and Kim et al. reported similar cases in which rapid an-

ABBREVIATIONS ACA = anterior cerebral artery; DWI = diffusion-weighted imaging; GEE = generalized estimating equation; ICA = internal carotid artery; MCA = middle cerebral artery; MRA = MR angiography; $\mathrm{mRS}=$ modified Rankin Scale; PCA = posterior cerebral artery; STA = superficial temporal artery; TIA = transient ischemic attack. SUBMITTED January 31, 2014. ACCEPTED October 21, 2014.

INCLUDE WHEN CITING Published online November 28, 2014; DOI: 10.3171/2014.10.JNS14231.

DISCLOSURE The authors report no conflict of interest concerning the materials or methods used in this study or the findings specified in this paper. 
giographic progression resulted in fatal outcome. ${ }^{4,18} \mathrm{Kim}$ et al. reported that younger patients were more likely to suffer from an aggressive clinical course, of whom a substantial number experienced repeat stroke. ${ }^{17}$

While the impact of such unstable disease on clinical outcome is receiving more focused attention, few studies have attempted to clearly define this condition. Accurate risk stratification in moyamoya disease requires objective definition of disease instability. According to the articles mentioned above, ${ }^{4,17,18}$ disease progression and repeat stroke are considered essential factors reflecting instability of moyamoya disease. The concept of unstable moyamoya disease defined as either rapid disease progression or repeat stroke seems reasonable, considering that unstable angina pectoris, the concept representing instability of angina pectoris, is also characterized as an exacerbating or recurrent symptom with rapid progression of stenosis in the coronary artery. ${ }^{1,23,27}$

Treatment of unstable moyamoya disease is challenging because patients with the disease commonly develop stroke during the perioperative period or even while awaiting surgery. ${ }^{7,17}$ Direct bypass, such as superficial temporal artery-to-middle cerebral artery (STA-MCA) bypass, has the advantage of contributing to increased cerebral blood flow soon after surgery. ${ }^{10}$ The safety and efficacy of direct bypass for unstable disease are, however, controversial.,17

This retrospective cohort study had 2 objectives: to detect clinical factors associated with unstable moyamoya disease and to determine whether the presence of such instability is associated with postoperative silent or symptomatic ischemic lesions detected on diffusion-weighted imaging (DWI).

\section{Methods}

This study was approved by the ethics committee of the Kyoto University Graduate School of Medicine.

\section{Selection Criteria}

A total of 88 pediatric and adult patients with moyamoya disease underwent 148 revascularization surgeries at Kyoto University Hospital between 2009 and 2013. The inclusion criteria for the present study were as follows: diagnosis of moyamoya disease or moyamoya syndrome; patients who underwent direct revascularization at Kyoto University Hospital in 2009 or thereafter; and patients who underwent routine postoperative DWI no longer than 14 days after surgery.

Diagnoses were made according to the criteria proposed by the Research Committee on Moyamoya Disease in Japan. "Moyamoya syndrome" is defined as a secondary moyamoya phenomenon caused by an underlying disease such as an autoimmune disease, meningitis, brain tumor, hyperthyroidism, Down syndrome, neurofibromatosis Type 1 , or a history of head irradiation. ${ }^{24}$ The present study also included unilateral disease in which only 1 side of the ICA was involved. "Direct bypass" was defined as a direct anastomotic procedure including STA-MCA, STA-anterior cerebral artery (ACA), and occipital arteryposterior cerebral artery (PCA) bypasses.

Of the 148 consecutive revascularization procedures, 3 were excluded because they involved only indirect bypass in the ACA territory. Although postoperative MRI with DWI was mandatory in our treatment protocol, postoperative DWI was not performed after 5 procedures (these cases were excluded from the study) because a postoperative MRI scan was performed but no DWI was acquired (4 procedures), and because no MRI was performed since the patient sought early discharge (1 procedure). None of these 5 patients presented with a new neurological deficit after surgery. Consequently, 140 procedures conducted in 86 patients were included in the present study (Fig. 1).

\section{Variables}

Unstable moyamoya disease, a primary variable of interest, was defined as a condition with evidence of either rapid stenosis progression or repeated stroke. "Rapid stenosis progression" was defined as progression of a steno-occlusive lesion in an ICA, ACA, MCA, or PCA that had occurred within 6 months (Fig. 2). Whether the progression had occurred was determined using an MR angiography (MRA) scoring system, ${ }^{8}$ and the scores were compared between 2 different sessions performed before surgery. Almost all patients referred to our institution had already undergone MRA, the results of which could be used as control imaging. If only 1 imaging session had been performed before surgery, or if the interval between the 2 sessions was less than 2 weeks, the data were eliminated from further analysis. Decreased antegrade flow due to bypass in the ipsilateral MCA territory was not regarded as stenosis progression. "Repeat stroke" was defined as newly developed symptomatic infarctions confirmed on DWI and occurring at least twice during an interval not exceeding 6 months.

The other variables possibly affecting surgical outcome were identified from previous literature: age younger than 3 years, ${ }^{16}$ female sex, ${ }^{15}$ presence of underlying disease causing moyamoya syndrome, ${ }^{6,22}$ transient ischemic attacks (TIAs) occurring at a frequency exceeding 3 times per month, ${ }^{16}$ radiographic evidence of preexisting infarc-

\section{8 patients (148 procedures)}

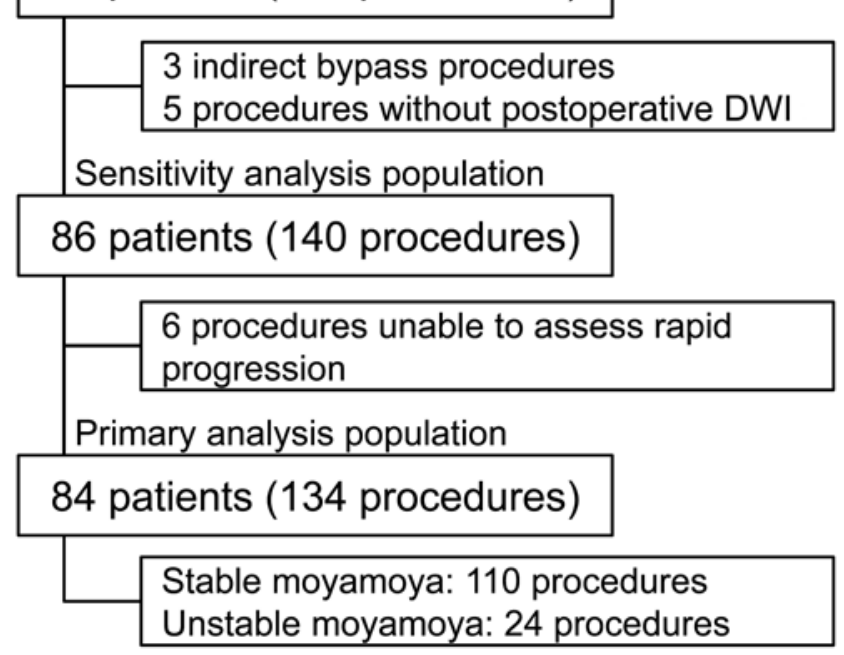

FIG. 1. Flowchart for patient inclusion. 

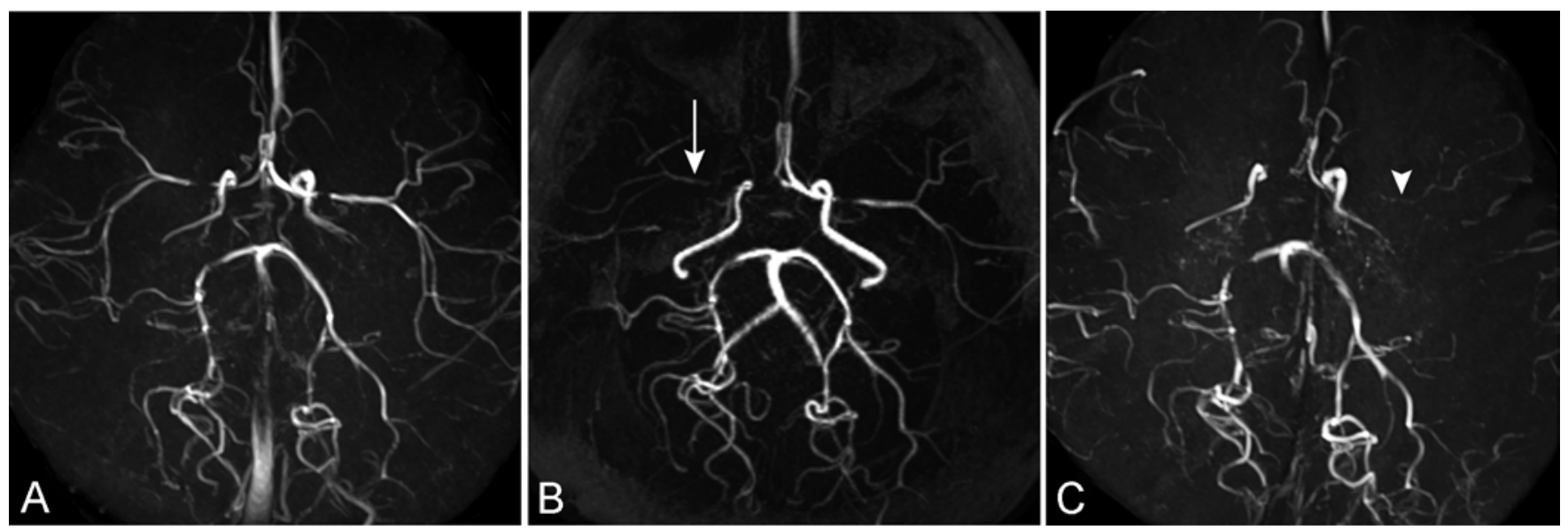

FIG. 2. Serial MRA images obtained in a 1-year-old boy representing rapid disease progression. A: Image obtained 2 months before referral to our hospital, revealing moderate stenosis in the terminal portion of the ICA and $M_{1}$ segment of the MCA bilaterally. B: Image obtained at admission to our hospital, revealing signal decrease in the right MCA compared with that obtained 2 months previously, suggesting rapid disease progression in the right MCA. The patient subsequently underwent direct bypass for the right MCA territory. C: Image obtained 2 months after surgery, revealing an invisible signal in the left MCA, suggesting rapid disease progression in the left MCA.

tion, $, 16,25$ recent stroke occurring no more than 6 weeks before surgery or evidence of recent stroke on preoperative DWI,, 16 advanced stage moyamoya (Suzuki Stages IV, V, and VI) in the ICA., ${ }^{11}$ and disease involvement in the PCA. ${ }^{11}$ "Unilateral disease" was defined as unilateral stenosis or occlusion of the terminal portion of the ICA with the formation of moyamoya vessels accompanied by no lesion or a subtle lesion around the contralateral terminal portion of the ICA. "Severe hemodynamic compromise" was defined as a defect in the cerebral blood flow both at rest and after acetazolamide challenge revealed on SPECT. Administration of antiplatelet agents was also recorded.

\section{Surgery}

All patients underwent MRI, SPECT, and angiography before surgery. Surgical revascularization was indicated for patients with cerebral ischemic manifestation. Patients presenting with other symptoms, such as epilepsy and intracranial hemorrhage, were also considered candidates for surgical revascularization as long as hemodynamic compromise existed. All patients underwent direct bypass, STA-MCA anastomosis, or combined bypass comprising STA-MCA anastomosis and encephalo-myo-synangiosis as a first-line treatment. ${ }^{13}$ After completing 2 sessions of revascularization for the MCA territories, some patients required additional revascularization surgeries for the ACA and PCA territories. Our surgical procedures and treatment protocol are described in previous literature. ${ }^{5,12,13}$ Antiplatelet agents, if administered, were discontinued for 3 days before surgery and 2 days after surgery.

During general anesthesia, $\mathrm{PaCO}_{2}$ and end-tidal $\mathrm{CO}_{2}$ were closely monitored, and the level of $\mathrm{PaCO}_{2}$ was strictly maintained between 37 and $40 \mathrm{~mm} \mathrm{Hg}$.

\section{Outcome}

All silent and symptomatic ischemic lesions, a primary outcome in this study, were diagnosed by neuroradi- ologists based on the results of DWI performed no longer than 14 days after surgery. A symptomatic lesion was defined as one causing a decline in the modified Rankin Scale (mRS) score. Such lesions were classified into either of 2 categories: cortical infarction or subcortical infarction. The patency of the bypass was also assessed using MRA performed in the same imaging session.

\section{Statistical Analysis}

Since more than half of the patients underwent multiple procedures, postoperative outcomes for such patients might not be statistically independent. In consideration of these dependencies, we used the generalized estimating equation (GEE) approach for the demographic descriptions of stable and unstable moyamoya disease as well as univariate and multivariate logistic regression analyses. ${ }^{2}$ All $p$ values and confidence intervals were calculated with robust standard error estimates from the GEE approach with the independent working correlation structure.

Because of rare outcome events, we selected preoperative variables only with $\mathrm{p}$ values $<0.05$ as covariates adjusted for the multivariate GEE logistic analysis. In light of its clinical importance, radiographic evidence of infarction was incorporated into the multivariate analysis regardless of its $\mathrm{p}$ value. As mentioned in Results, assessment of disease progression was not possible for 6 procedures; hence, these procedures were excluded from the primary analysis. We also conducted a sensitivity analysis that included the 6 procedures, all of which were assumed to be those of unstable disease (Fig. 1), considering the possibility that such patients might undergo only 1 MRA session because of rapid progression. We fit the same GEE logistic regression for both the primary and sensitivity analyses. Two-sided values of $\mathrm{p}<0.05$ and $95 \%$ confidence intervals of odds ratios that do not include 1 were considered significant. All analyses were performed using JMP (version 9) software and Windows SAS (version 9.3, SAS Institute Inc.). 


\section{Results}

\section{Characteristics of Unstable Disease}

Demographics of the 86 patients are summarized in Table 1. Among the 140 procedures performed in these patients, 6 procedures could not be assessed for disease progression for the following reasons: 2 MRI sessions were performed before surgery, but the interval was less than 2 weeks ( 3 procedures); only 1 MRI session was performed before surgery because the patient had suffered a minor stroke during admission to the neurological department at our hospital and was promptly referred to a neurosurgeon (1 procedure); and the surgeon did not require a second MRI before surgery because a baseline MRI had been performed shortly before referral (2 procedures). Postoperative DWI revealed neither silent nor symptomatic lesions in these 6 cases. Baseline characteristics of the 134 included and 6 excluded cases were compared, and no variable significantly differed between the 2 groups except for the prevalence of recent stroke $(9 \%$ in the included group and $50 \%$ in the excluded group, $\mathrm{p}=0.017$ ).

Of the 134 procedures included in the primary analysis, $24(17.9 \%)$ were classified as unstable disease (Table 2): 16 as rapid progression, 6 as repeat stroke, and 2 as both. Disease progression at an interval exceeding 6 months had occurred before 5 procedures, all of which were classified in the stable group.

Table 3 summarizes baseline characteristics of the stable and unstable groups. Compared with the stable group, the unstable group included more patients younger than 3 years $(p=0.029)$, more completed stroke as an initial manifestation $(p<0.001)$, more evidence of infarction ( $p$ $=0.030$ ), and more underlying disease causing moyamoya syndrome $(\mathrm{p}=0.049)$. The median interval between the 2 MRA sessions was 90.5 days (range 14-352 days) for the stable group and 81.5 days (range $27-175$ days) for the unstable group.

TABLE 1. Demographic and clinical characteristics of 86 patients included in the study

\begin{tabular}{cc}
\hline \multicolumn{1}{c}{ Variable } & Value $^{*}$ \\
\hline Median age (yrs, IQR) & $18.5(7.75-39.25)$ \\
\hline Female & $56(65.1)$ \\
\hline Initial manifestation & $51(59.3)$ \\
\hline TIA & $18(20.9)$ \\
\hline Completed stroke & $11(12.8)$ \\
\hline Intracranial hemorrhage & $3(3.5)$ \\
\hline Epilepsy & $3(3.5)$ \\
\hline Asymptomatic & $7(8.1)$ \\
\hline Underlying disease & \\
\hline No. of procedures & $37(43.0)$ \\
\hline 1 & $44(51.2)$ \\
\hline 2 & $5(5.8)$ \\
\hline 3 &
\end{tabular}

$\mathrm{IQR}=$ interquartile range.

* Values are presented as the number of patients (\%) unless noted otherwise.
TABLE 2. Details of classification of 134 procedures as stable or unstable disease

\begin{tabular}{lc}
\hline \multicolumn{1}{c}{ Variable } & No. of Procedures \\
\hline Stable disease (110 procedures) & \\
\hline Neither progression nor repeated stroke & 105 \\
\hline Progression over $>6$ mos & 5 \\
\hline Unstable disease (24 procedures) & \\
\hline Rapid progression w/in 6 mos & 16 \\
\hline Repeated stroke w/in 6 mos & 6 \\
\hline Both rapid progression \& repeated stroke & 2 \\
\hline
\end{tabular}

\section{Overall Outcome}

Postoperative DWI-defined lesions were detected after $13(9.3 \%)$ of 140 procedures (Fig. 3). Six lesions were clinically silent, while 7 caused transient or permanent symptoms. A permanent ischemic complication occurred in 4 procedures $(2.9 \%)$. These symptomatic lesions, all of which occurred in the unstable disease group, represented a cortical infarction remotely located from the anastomosis site, while the remaining 9 lesions included cortical and subcortical infarctions (Fig. 3). Lesions on the side contralateral to the surgery were detected on DWI in 3 procedures. The patency of all bypasses was confirmed using MRA.

For patients who suffered a permanent ischemic complication, at the time of discharge 1 patient had an mRS score of 1 , and 3 patients had an mRS score of 2 . At the time of last follow-up, 2 of these patients had an mRS score of 1 , and 2 patients had an mRS score of 2 .

\section{Association Between Unstable Disease and Postoperative DWI-Detected Lesions}

The incidence of postoperative DWI-detected lesions in the unstable disease group was 33.3\% (8 of 24 procedures), which was significantly higher than that in the stable disease group $(5 / 110$ procedures [4.5\%], $\mathrm{p}<0.001)$. Infarction causing permanent morbidity was seen after 4 procedures $(16.7 \%)$ in the unstable disease group, while no permanent morbidity was observed in the stable disease group. No stroke occurred after the 14th postoperative day in either group.

For the univariate analysis, unstable disease $(\mathrm{p}<0.001)$, underlying disease $(\mathrm{p}=0.028)$, and recent stroke $(\mathrm{p}=$ 0.012 ) were identified as preoperative variables associated with postoperative DWI-detected lesions (Table 4). The primary analysis revealed that unstable disease was a statistically significant factor independently associated with DWI-detected lesions (OR 6.62 [95\% CI 1.79-24.5]; Table $5)$. Unstable disease remains statistically significant in the sensitivity analysis (OR 5.36 [95\% CI 1.47-19.6]).

\section{Discussion}

The results of the present study suggest that age younger than 3 years and underlying disease causing moyamoya syndrome are associated with unstable moyamoya disease. Our results indicate that, despite the relatively low overall ischemic complication rate (2.9\%), unstable disease is an 
TABLE 3. Demographic descriptions of stable and unstable moyamoya disease*

\begin{tabular}{|c|c|c|c|}
\hline Variable & Stable (110 procedures) & Unstable (24 procedures) & $p$ Value \\
\hline \multicolumn{4}{|l|}{ Age in yrs } \\
\hline Mean \pm SD & $21.3 \pm 15.1$ & $18.0 \pm 18.5$ & 0.461 \\
\hline Median (range) & $17.5(0-52)$ & $7.5(1-51)$ & \\
\hline Age $<3$ yrs & $3(2.7)$ & $4(16.7)$ & 0.029 \\
\hline Female & $77(70.0)$ & $13(54.2)$ & 0.184 \\
\hline Mean systolic BP in mm Hg & $120.2 \pm 15.2$ & $120.7 \pm 19.9$ & 0.926 \\
\hline Mean diastolic BP in $\mathrm{mm} \mathrm{Hg}$ & $73.2 \pm 15.6$ & $75.4 \pm 17.0$ & 0.620 \\
\hline \multicolumn{4}{|l|}{ Initial manifestation } \\
\hline Completed stroke & $16(14.5)$ & $13(54.2)$ & $<0.001$ \\
\hline TIA & $74(67.3)$ & $4(16.7)$ & $<0.001$ \\
\hline Intracranial hemorrhage & $13(11.8)$ & $3(12.5)$ & 0.943 \\
\hline Other & $7(6.4)$ & $4(16.7)$ & \\
\hline Underlying disease & $7(6.4)$ & $5(20.8)$ & 0.049 \\
\hline Frequency of TIA >3 times/mo & $8(7.3)$ & $3(12.5)$ & 0.339 \\
\hline Radiographic evidence of infarction & $66(60)$ & $20(83.3)$ & 0.030 \\
\hline Recent stroke & $5(4.5)$ & $7(29.2)$ & $<0.001$ \\
\hline Unilateral disease & $11(10.0)$ & $3(12.5)$ & 0.713 \\
\hline \multicolumn{4}{|l|}{ Suzuki stage } \\
\hline I & $5(4.5)$ & $1(4.2)$ & \\
\hline II & $32(29.1)$ & $8(33.3)$ & \\
\hline III & $59(53.6)$ & $14(58.3)$ & \\
\hline IV & $14(12.7)$ & $1(4.2)$ & \\
\hline V & 0 & 0 & \\
\hline Suzuki Stage $\geq I V$ & $14(12.7)$ & $1(4.2)$ & 0.270 \\
\hline PCA involvement & $32(29.1)$ & $10(41.7)$ & 0.283 \\
\hline Severe hemodynamic compromise $\neq$ & $72(66.7)$ & $18(75.0)$ & 0.412 \\
\hline Antiplatelet agent administration & $92(83.6)$ & $21(87.5)$ & 0.709 \\
\hline Median MRI interval in days (range) & $90.5(14-352)$ & $81.5(27-175)$ & 0.048 \\
\hline
\end{tabular}

independent risk factor for perioperative DWI-detected lesions.

The incidence of disease progression in moyamoya disease, which is receiving more attention especially regarding cases of unilateral disease, is not particularly rare. Disease progression occurs in 54\% of pediatric patients over a mean period of 5.4 years and in $23.8 \%$ of adult patients over a mean period of 6.1 years. ${ }^{19,21}$ A more recent study estimated the 3-year cumulative incidence of disease progression as $19 \%$ in children and adolescents with unilateral disease. ${ }^{28}$ Younger age, ${ }^{26,28}$ female sex,${ }^{19}$ contralateral angiographic abnormality, ${ }^{20,26}$ and underlying cause of moyamoya syndrome ${ }^{21}$ have been considered risk factors for contralateral progression of unilateral disease. The results of our study, which found younger age and underlying disease as risk factors for unstable disease, are in line with these reports. The mean time to progression estimated in the previous studies ranged from 14.3 to 34 months. . $^{14,19,20}$ Our results may add important information to those of pre- vious studies, because more rapid disease progression was observed in a substantial number of patients.

Several factors, such as age younger than 3 years, ${ }^{16} \mathrm{fe}-$ male sex, ${ }^{15}$ presence of underlying disease, ${ }^{6,22}$ frequent TIAs, ${ }^{16}$ radiographic evidence of preexisting infarction, ${ }^{9,16,25}$ recent episode of stroke, ${ }^{9,16}$ advanced angiographic stage, ${ }^{11}$ and disease involvement in the PCA,${ }^{11}$ are believed to increase ischemic complications after bypass surgery for moyamoya disease. Although young age and preexisting infarction were generally considered major risk factors for surgical outcome in moyamoya disease, some controversy remains in the literature regarding what constitutes a risk factor. Our univariate result, indicating that underlying disease and recent stroke are significant, partly coincides with the literature. In addition, our multivariate results suggest that unstable disease is another independent risk factor for perioperative ischemic complications.

On the other hand, our results did not reveal a statistically significant impact of preexisting infarction on outcome, 

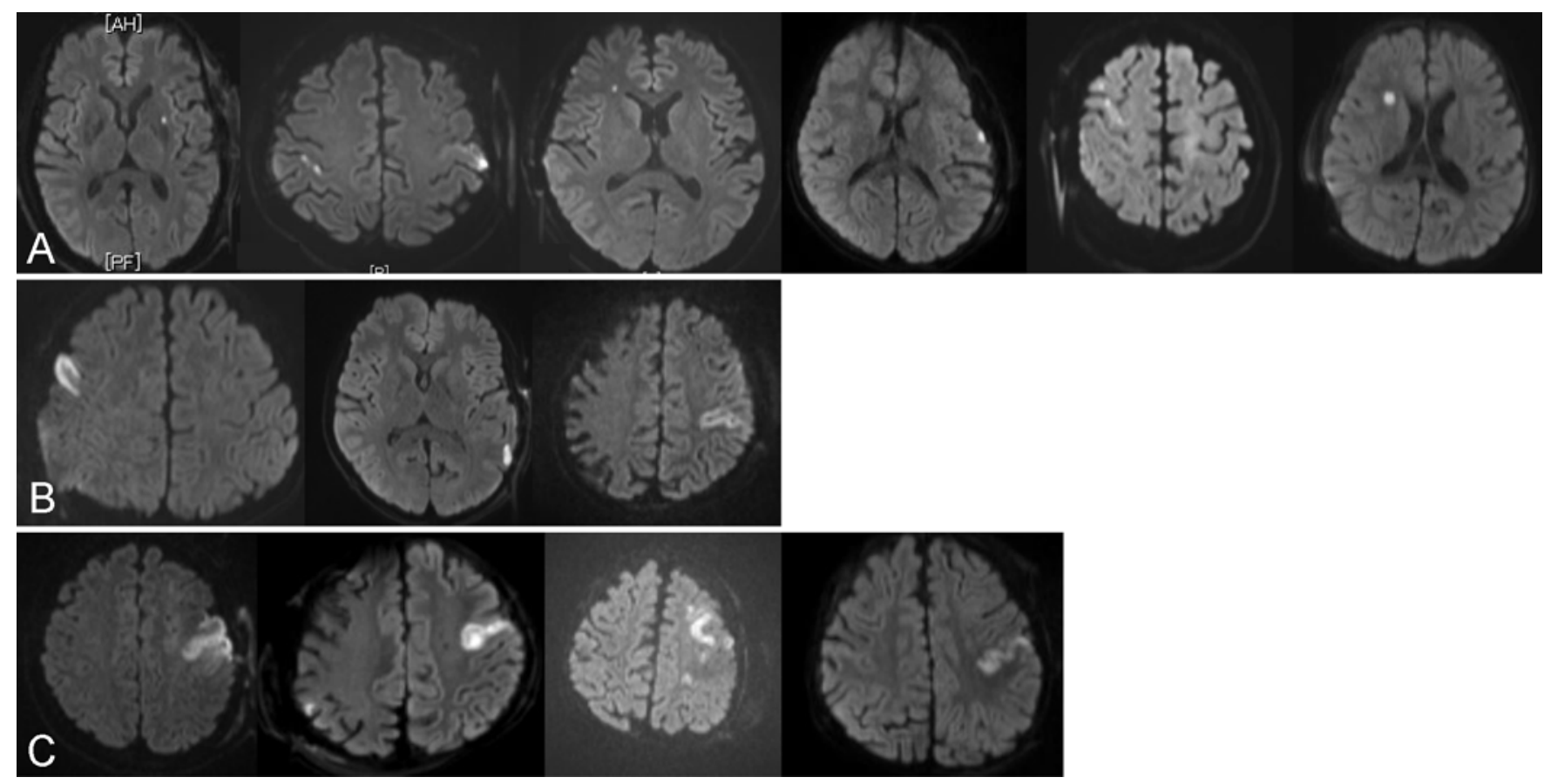

FIG. 3. Lesions detected on postoperative DWI. A: Silent lesions. B: Lesions causing transient symptoms. C: Lesions causing permanent symptoms.

which contradicts findings of some previous reports., ${ }^{9,16,25} \mathrm{~A}$ possible explanation for this contradiction is that unstable disease, including infarct as defined, acted as an intervening variable in multivariate analysis, which might diminish the true effect of infarct on outcome. A relatively high odds ratio was observed for preexisting infarct, and a study with a larger sample size may have detected its statistically significant impact on perioperative ischemic complication. Another reason we did not find a significant effect from

TABLE 4. Univariate analyses of putative factors and postoperative DWI-detected lesions

\begin{tabular}{lcc}
\hline \multicolumn{1}{c}{ Variable } & OR $(95 \% \mathrm{Cl})$ & $\mathrm{p} \mathrm{Value}$ \\
\hline Unstable moyamoya & $10.5(2.69-41.0)$ & $<0.001$ \\
\hline Preop factors & & \\
\hline Age $(\mathrm{yrs})$ & $0.99(0.94-1.05)$ & 0.792 \\
\hline Age $<3$ yrs & $4.22(0.39-46.1)$ & 0.238 \\
\hline Female & $0.53(0.13-2.16)$ & 0.379 \\
\hline Systolic BP & $1.02(0.97-1.07)$ & 0.422 \\
\hline Diastolic BP & $1.02(0.97-1.08)$ & 0.360 \\
\hline Underlying disease & $6.28(1.22-32.4)$ & 0.028 \\
\hline Frequency of TIAs $>3$ times/mo & $2.26(0.43-12.0)$ & 0.336 \\
\hline Radiographic evidence of infarction & $7.62(0.88-65.7)$ & 0.065 \\
\hline Recent stroke & $6.28(1.51-26.1)$ & 0.012 \\
\hline Unilateral disease & $0.69(0.08-6.11)$ & 0.741 \\
\hline Suzuki Stage $\geq$ IV & $2.10(0.29-7.89)$ & 0.625 \\
\hline PCA involvement & $0.97(0.22-4.26)$ & 0.969 \\
\hline Severe hemodynamic compromise* & $6.31(0.90-44.2)$ & 0.064 \\
\hline Antiplatelet agent administration & $2.38(0.27-21.0)$ & 0.436 \\
\hline * Data we unailab
\end{tabular}

\footnotetext{
* Data were unavailable for 2 procedures.
}

preexisting infarct might be a difference in surgical procedures. The prestigious study by Kim et al., concluding that age younger than 3 years and preexisting infarct were risk factors for surgical ischemic complication, addressed indirect bypass. ${ }^{16}$ On the other hand, Guzman et al., analyzing the outcomes of 450 direct bypasses, found no association between age and perioperative ischemia. ${ }^{6}$

One possible mechanism of infarction developing after bypass surgery in the unstable disease group could be hemodynamic shift after direct bypass. Hayashi et al. discussed the mechanism of postoperative focal hypoperfusion detected by SPECT? They speculated that the reversed MCA flow induced by the bypass graft could conflict with the original MCA flow, resulting in relative hypoperfusion in the remote territory of the MCA. Such a hemodynamic shift may be more prevalent in unstable disease. In unstable disease, leptomeningeal collateral flow may fail to develop sufficiently because of rapid disease progression. As a result, the bypass flow may conflict more strongly with the original antegrade flow supplying the MCA territory. Our finding that all symptomatic lesions occurring in unstable disease represent a cortical infarction remotely located from the anastomosis site may support this speculation.

Our study has several limitations. First, the study excluded from the primary analysis 6 procedures before which only 1 session of MRA had been performed. The impact of possible bias caused by this exclusion may be minimal, however, because the sensitivity analysis also revealed statistical significance of unstable disease. Second, the retrospective study design meant that the interval between the 2 MRA sessions and the modality of MRA varied among patients. This might have caused a selection bias regarding the classification of unstable disease. Third, the result of 
TABLE 5. Estimated ORs and $95 \% \mathrm{Cls}$ for primary and sensitivity multivariate analyses

\begin{tabular}{|c|c|c|c|c|}
\hline \multirow[b]{2}{*}{ Variable } & \multicolumn{2}{|c|}{ Primary Analysis } & \multicolumn{2}{|c|}{ Sensitivity Analysis } \\
\hline & OR $(95 \% \mathrm{Cl})$ & p Value & OR $(95 \% \mathrm{Cl})$ & $\mathrm{p}$ Value \\
\hline Unstable moyamoya & $6.62(1.79-24.5)$ & 0.005 & $5.36(1.47-19.6)$ & 0.011 \\
\hline Underlying disease & $2.98(0.56-15.8)$ & 0.200 & $3.68(0.72-18.8)$ & 0.117 \\
\hline Radiographic evidence of infarction & $3.75(0.39-36.3)$ & 0.254 & $4.07(0.42-39.9)$ & 0.228 \\
\hline Recent stroke & $1.88(0.58-6.09)$ & 0.295 & $1.36(0.44-4.21)$ & 0.593 \\
\hline
\end{tabular}

the multivariate analysis has a relatively large confidence interval, which is attributable to the small number of cases. Larger prospective studies are therefore necessary to confirm the significant impact of unstable moyamoya disease on perioperative ischemic complications. These studies might also reveal the statistical significance of other variables such as preexisting infarct.

Considering the devastating nature of unstable moyamoya disease reported to date, $,, 17,18$ early surgical revascularization might be recommended. Kim et al. similarly stressed the importance of early surgical intervention for aggressive moyamoya disease observed in children. ${ }^{17} \mathrm{On}$ the other hand, our finding, which detected the unstable state as an independent surgical risk factor, shed light on the need for preoperative diagnosis and specific perioperative management of unstable moyamoya disease. In terms of the diagnosis, performing MRA immediately before surgery might be useful, as the disease progression could be detected through a comparison with previous findings. In terms of treatment, perioperative management should be focused more on preventing ischemic complications. Although intentional lowering of blood pressure is considered for patients with hyperperfusion complications, ${ }^{3}$ those with unstable disease should instead be maintained in a normotensive or slightly hypertensive state. Strict control of blood $\mathrm{CO}_{2}$ level during surgery, in which $\mathrm{PaCO}_{2}$ is maintained between 37 and $40 \mathrm{~mm} \mathrm{Hg}$ at our institution, should also be undertaken. More aggressive use of antiplatelet agents could be adopted as a possible option for unstable disease. Although we adopted direct bypass as the first choice for treating moyamoya disease, tentative indirect bypass followed by elective direct bypass may be considered for unstable disease. This strategy can be indicated especially when acute hemorrhagic infarction after direct bypass as well as hemodynamic shift is concerned. The efficacy of these possible methods of management of unstable disease should be tested in future studies.

\section{Conclusions}

Unstable moyamoya disease, reasonably defined as cases of rapid progression or repeated stroke, represents a clinically challenging condition. It is more prevalent in patients younger than 3 years and those with underlying disease causing moyamoya syndrome. Unstable moyamoya disease is a possible risk factor associated with perioperative ischemic complication. The concept of unstable moyamoya disease may contribute to further improvement in the surgical results of moyamoya disease as a result of focused perioperative management arising from appropriate surgical risk stratification.

\section{Acknowledgments}

We are grateful to Dr. Tosiya Sato, Professor of the Department of Biostatics, Kyoto University School of Public Health, for providing advice on statistics and performing the GEE analyses. We also appreciate the efforts of Dr. Noritaka Sano, Ms. Yuko Tanaka, and Ms. Yuka Aramaki in collecting patient records.

\section{References}

1. Austen WG, Edwards JE, Frye RL, Gensini GG, Gott VL, Griffith LS, et al: A reporting system on patients evaluated for coronary artery disease. Report of the Ad Hoc Committee for Grading of Coronary Artery Disease, Council on Cardiovascular Surgery, American Heart Association. Circulation 51 (4 Suppl):5-40, 1975

2. Diggle P, Heagerty PJ, Liang KY, Zeger SL: Analysis of Longitudinal Data, ed 2. Oxford, UK: Oxford University Press, 2002

3. Fujimura M, Inoue T, Shimizu H, Saito A, Mugikura S, Tominaga T: Efficacy of prophylactic blood pressure lowering according to a standardized postoperative management protocol to prevent symptomatic cerebral hyperperfusion after direct revascularization surgery for moyamoya disease. Cerebrovasc Dis 33:436-445, 2012

4. Fujiwara F, Yamada H, Hayashi S, Tamaki N: [A case of adult moyamoya disease showing fulminant clinical course associated with progression from unilateral to bilateral involvement.] No Shinkei Geka 25:79-84, 1997 (Jpn)

5. Funaki T, Takahashi JC, Takagi Y, Yoshida K, Araki Y, Kikuchi T, et al: Impact of posterior cerebral artery involvement on long-term clinical and social outcome of pediatric moyamoya disease. J Neurosurg Pediatr 12:626-632, 2013

6. Guzman R, Lee M, Achrol A, Bell-Stephens T, Kelly M, Do $\mathrm{HM}$, et al: Clinical outcome after 450 revascularization procedures for moyamoya disease. Clinical article. J Neurosurg 111:927-935, 2009

7. Hayashi T, Shirane R, Fujimura M, Tominaga T: Postoperative neurological deterioration in pediatric moyamoya disease: watershed shift and hyperperfusion. J Neurosurg Pediatr 6:73-81, 2010

8. Houkin K, Nakayama N, Kuroda S, Nonaka T, Shonai T, Yoshimoto T: Novel magnetic resonance angiography stage grading for moyamoya disease. Cerebrovasc Dis 20:347354,2005

9. Hyun SJ, Kim JS, Hong SC: Prognostic factors associated with perioperative ischemic complications in adult-onset moyamoya disease. Acta Neurochir (Wien) 152:1181-1188, 2010

10. Ishikawa T, Houkin K, Kamiyama H, Abe H: Effects of surgical revascularization on outcome of patients with pediatric moyamoya disease. Stroke 28:1170-1173, 1997

11. Jung YJ, Ahn JS, Kwon H, Kwun BD: Ischemic complications occurring in the contralateral hemisphere after surgical treatment of adults with moyamoya disease. J Korean Neurosurg Soc 50:492-496, 2011

12. Karasawa J, Kikuchi H, Furuse S, Kawamura J, Sakaki T: Treatment of moyamoya disease with STA-MCA anastomosis. J Neurosurg 49:679-688, 1978 
13. Karasawa J, Kikuchi H, Furuse S, Sakaki T, Yoshida Y: A surgical treatment of "moyamoya" disease "encephalo-myo synangiosis." Neurol Med Chir (Tokyo) 17:29-37, 1977

14. Kelly ME, Bell-Stephens TE, Marks MP, Do HM, Steinberg GK: Progression of unilateral moyamoya disease: a clinical series. Cerebrovasc Dis 22:109-115, 2006

15. Khan N, Achrol AS, Guzman R, Burns TC, Dodd R, BellStephens T, et al: Sex differences in clinical presentation and treatment outcomes in Moyamoya disease. Neurosurgery 71:587-593, 2012

16. Kim SH, Choi JU, Yang KH, Kim TG, Kim DS: Risk factors for postoperative ischemic complications in patients with moyamoya disease. J Neurosurg 103 (5 Suppl):433-438, 2005

17. Kim SK, Seol HJ, Cho BK, Hwang YS, Lee DS, Wang KC: Moyamoya disease among young patients: its aggressive clinical course and the role of active surgical treatment. Neurosurgery 54:840-846, 2004

18. Kim TW, Seo BR, Kim JH, Kim YO: Rapid progression of unilateral moyamoya disease. J Korean Neurosurg Soc 49:65-67, 2011

19. Kuroda S, Ishikawa T, Houkin K, Nanba R, Hokari M, Iwasaki Y: Incidence and clinical features of disease progression in adult moyamoya disease. Stroke 36:2148-2153, 2005

20. Lee SC, Jeon JS, Kim JE, Chung YS, Ahn JH, Cho WS, et al: Contralateral progression and its risk factor in surgically treated unilateral adult moyamoya disease with a review of pertinent literature. Acta Neurochir (Wien) 156:103-111, 2014

21. Lin N, Baird L, Koss M, Kopecky KE, Gone E, Ullrich NJ, et al: Discovery of asymptomatic moyamoya arteriopathy in pediatric syndromic populations: radiographic and clinical progression. Neurosurg Focus 31(6):E6, 2011

22. Mallory GW, Bower RS, Nwojo ME, Taussky P, Wetjen NM, Varzoni TC, et al: Surgical outcomes and predictors of stroke in a North American white and African American moyamoya population. Neurosurgery 73:984-992, 2013

23. Moise A, Théroux P, Taeymans Y, Descoings B, Lespérance
J, Waters DD, et al: Unstable angina and progression of coronary atherosclerosis. N Engl J Med 309:685-689, 1983

24. Research Committee on the Pathology and Treatment of Spontaneous Occlusion of the Circle of Willis: Guidelines for diagnosis and treatment of moyamoya disease (spontaneous occlusion of the circle of Willis). Neurol Med Chir (Tokyo) 52:245-266, 2012

25. Sato K, Shirane R, Yoshimoto T: Perioperative factors related to the development of ischemic complications in patients with moyamoya disease. Childs Nerv Syst 13:68-72, 1997

26. Smith ER, Scott RM: Progression of disease in unilateral moyamoya syndrome. Neurosurg Focus 24(2):E17, 2008

27. Yeghiazarians Y, Braunstein JB, Askari A, Stone PH: Unstable angina pectoris. N Engl J Med 342:101-114, 2000

28. Yeon JY, Shin HJ, Kong DS, Seol HJ, Kim JS, Hong SC, et al: The prediction of contralateral progression in children and adolescents with unilateral moyamoya disease. Stroke 42:2973-2976, 2011

\section{Author Contributions}

Conception and design: Funaki. Acquisition of data: Funaki. Analysis and interpretation of data: Funaki, Okada, Fushimi. Drafting the article: Funaki. Critically revising the article: Takahashi, Takagi, Mitsuhara, Miyamoto. Reviewed submitted version of manuscript: Takahashi, Takagi, Kikuchi, Yoshida, Mitsuhara, Kataoka, Okada, Fushimi, Miyamoto. Approved the final version of the manuscript on behalf of all authors: Funaki. Statistical analysis: Funaki. Study supervision: Takahashi, Takagi, Kikuchi, Yoshida, Kataoka, Miyamoto.

\section{Correspondence}

Takeshi Funaki, Department of Neurosurgery, Kyoto University Graduate School of Medicine, 54 Kawahara-cho, Shogoin, Sakyo-ku, Kyoto 606-8507, Japan. email: tfunaki@kuhp.kyoto-u. ac.jp. 